\title{
Design of Low Power 4-bit ALU Using Adiabatic Logic
}

\author{
Sriraj Dheeraj Turaga ${ }^{1}$, Kundan Vanama ${ }^{2}$, Rithwik Reddy Gunnuthula ${ }^{3}$ and \\ K.Jaya Datta Sai ${ }^{4}$ \\ ${ }^{1,2,3,4}$ (Department of Electronics and Communication, GITAM University - Hyderabad)
}

\begin{abstract}
This paper presents the implementation of a 4-bit Arithmetic Logic Unit (ALU) using Complementary Energy Path Adiabatic Logic (CEPAL). This static adiabatic logic has proved its advantage through the minimization of the 1/2CVdd2 energy dissipation occurring every cycle. Firstly, the performance characteristics of CEPAL 4-to-1 multiplexer and full adder are compared against the conventional static CMOS logic counterpart to identify its adiabatic power advantage. Finally, A 4-bit Arithmetic Logic Unit (ALU) is implemented with both the technologies and comparisons have been made. The analysis is carried out using the industry standard EDA design environment using $250 \mathrm{~nm}$ technology libraries from Tanner. The results prove that the CEPAL 4-bit ALU is 55\% more power efficient than the CMOS 4-bit ALU at $100 \mathrm{MHz}$ and at $2.5 \mathrm{~V}$ operating voltage.
\end{abstract}

Keywords: Low Power; Static Adiabatic logic; Complementary Energy Path Adiabatic Logic (CEPAL); Power Clock(PC); Full Adder; Arithmetic Logic Unit (ALU); Multiplexer(MUX); Very Large Scale Integration(VLSI)

\section{INTRODUCTION}

With the advancement in technology it has become possible to scale down the electronics involved in numerous applications. The advantage being that the technology becomes more portable and its applications increase, the tradeoff being that the power levels utilized at that scaled down level induces other effects on the circuit, i.e the power dissipation as heat increases, the stability decreases, the performance decreases and so on. Numerous techniques have been suggested over the years to overcome these problems. Adiabatic logic is a promising alternative to CMOS in reducing the power dissipation without having to compromise with noise immunity and driving ability. Many adiabatic Logics have been presented in the literature [4]-[17] over the years, but the fundamental principle remains the same.

Energy recovery techniques and adiabatic logic topologies minimize energy dissipation by maintaining low voltage drop across conducting devices at all times. The undissipated energies related to the charges are recycled and is not dissipated as heat. Many of the energy recovery techniques have been presented in the literature. [1]- [3]

Most of the adiabatic circuits have dynamic circuit properties and have high switching activity. This property makes the implementation of this logic on larger circuits difficult. In addition, the requirements of multiphase and multi clock also make them unfavorable in terms of design complexity and area. [20,21]

Quasi static energy recovery logic (QSERL) shown in the literature [18,19] has been known to overcome the drawbacks of these dynamic adiabatic logics. Its simplicity, static nature and use of only two complementary clocks make it easy to design and apply in complex circuits than the dynamic circuits which use triangular or trapezoidal clocking scheme. In spite of these advantages, this logic suffers from the output floating node associated with alternate hold phases in operation. Although this floating node can be avoided by adding clocked feedback keeper, there is still significant power loss and the added area would restrict its application. [20,21]

Complementary Energy Path Adiabatic Logic (CEPAL) is proposed in this paper. Motivated by the design constrains of the QSERL, CEPAL is designed which inherits all the advantages of the static logic QSERL and also eliminates the hold phases of the QSERL thus increasing its robustness and throughput. $[20,21]$ This logic presents an effective alternative to conventional CMOS for the realization of low power electronics. The performance of CEPAL has been analyzed using excessive experiments. This paper presents the design and experimental evaluation of a 4- bit ALU and a 4-to-1 multiplexer. In addition to this, our paper contains the simultaneous comparisons of power consumption and transistor count of both CMOS and CEPAL technology. It was found that the CEPAL ALU was 55\% more power efficient compared to the CMOS ALU.

\section{COMPLEMENTARY ENERGY PATH ADIABATIC LOGIC}

The Structure and working of the CEPAL is shown in figure 1. [20,21] It consists of a pair of charging and discharging transistors. A pull up network and a pull down network.This logic makes use of two sinusoidal power clocks, PC and PCbar, which are complementary to each other. Let us understand the working of this 
structure. Assuming the initial Vout is low, with the P- network on and the N-network off, Vout follows the power clock PC or PCbar which ever swings to the high level. Since the power clock is sinusoid in nature it ramps down which makes the Vout to follow it and generating a floating node. This situation is however avoided by the complementary clock which swings high, thus eliminating this weak high signal and also eliminating the hold state seen in two phase clock operated circuits. Similarly the weak low signal is also eliminated by the complementary clock. [22]-[24]

Let us assume the other case in which Vout is high with the P-network on and the N-network is off. The Vout remains high unless and until there is a change in the input. CEPAL has two more diodes in comparison to quasi static energy recovery logic (QSERL) shown in the literature [18,19], but power dissipation due to these additional diodes is not very large as there is only one charging or discharging diodes turned on at an instant of time. Thus QSERL and CEPAL circuits have similar power consumption, however considerable improvements in area and power overheads can be achieved in CEPAL circuits because the keeper is used in QSERL circuits to avoid erroneous operation during hold phase. [20,21]

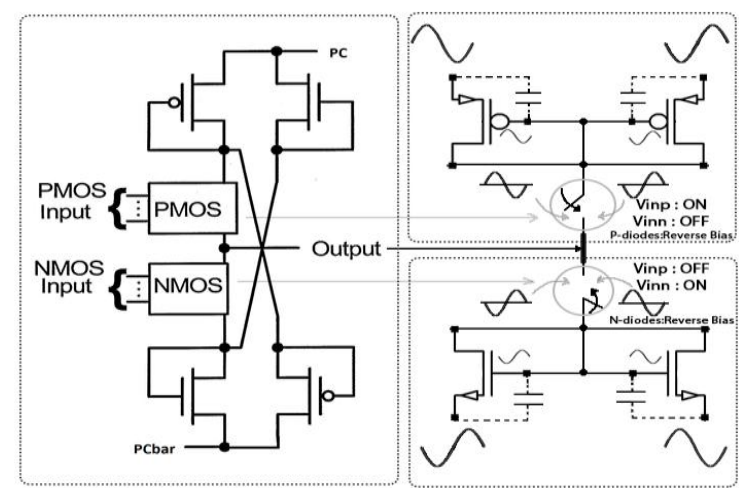

Figure 1: Complementary Energy Path Adiabatic Logic

\section{A. 4-to-1 Mux}

\section{DESIGN OF CEPAL 4-TO-1 MUX AND FULL ADDER}

The experimental work consists of simulating the circuit shown in Figure. 2. A MUX is a device used to handle multiple signals by selecting one of several input signals and forwarding it into a single line. This MUX is implemented using Tanner EDA with all the transistors of equal sizes of $\mathrm{W} / \mathrm{L}=0.35 \mu \mathrm{m} / 0.25 \mu \mathrm{m}$. The supply voltage and power clock frequency (PC) are $2.5 \mathrm{~V}$ and $100 \mathrm{MHz}$ respectively.

The number of transistors and the average power of the CMOS and the CEPAL for the MUX are shown in Table 1.

Table 1: MUX Transistor count \& Power Comparison

\begin{tabular}{|c|c|c|c|c|}
\hline Logic & P-MOS & N-MOS & Total Transistors & Average Power \\
\hline CMOS & 13 & 13 & 26 & $5.980 \mathrm{uW}$ \\
\hline CEPAL & 17 & 17 & 34 & $1.768 \mathrm{uW}$ \\
\hline
\end{tabular}

Though there is an increase in the number of transistors in the CEPAL technology, significant decrease in power can be seen. The CEPAL MUX is $70 \%$ more efficient compared to its CMOS counterpart.

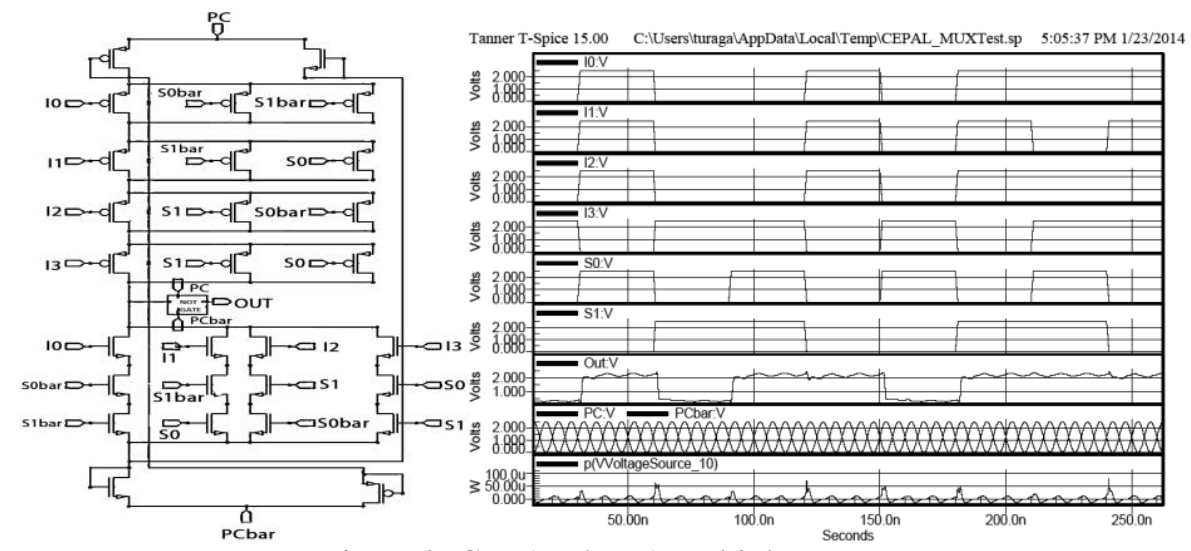

Figure 2: CEPAL 4-to-1 Multiplexer 
Figure 2 also shows the output waveforms of the CEPAL MUX. PC and PCbar are the two complementary power clock. The plot $\mathrm{p}$ (VVoltageSource_10) shows the power consumption of the power clock. The average of $\mathrm{p}$ (VVoltageSource_10)is given in table 1.

\section{B. Full Adder}

The Full Adder accepts three one bit inputs and generates a sum and carry output. The sum is generated by the XOR operation of all the inputs. This Full Adder is implemented here using the CEPAL technology. Figure 3 shows the schematic of the Full Adder which consists of forty five pMOS and forty five nMOS transistors. The number of transistors and the average power of the CMOS and the CEPAL for the Full Adder are shown in Table 2.

Table 2: Full Adder Transistor count \& Power Comparison

\begin{tabular}{|c|c|c|c|c|}
\hline Logic & P-MOS & N-MOS & Total Transistors & Average Power \\
\hline CMOS & 21 & 21 & 42 & $9.922 \mathrm{uW}$ \\
\hline CEPAL & 45 & 45 & 90 & $2.607 \mathrm{uW}$ \\
\hline
\end{tabular}

The CEPAL Full Adder is found to be $73 \%$ power efficient compared to CMOS technology this added advantage is a well worth tradeoff for the increased transistor count. Figure 3 shows the output waveforms of the CEPAL Full Adder. The logic ' 1 ' is $2.24 \mathrm{~V}$ and logic ' 0 ' is $0.31 \mathrm{~V}$. Plot PC and PCbar show the power clock given. The plot $\mathrm{p}$ (VVoltageSource_10) show the power consumption of the powerclock.

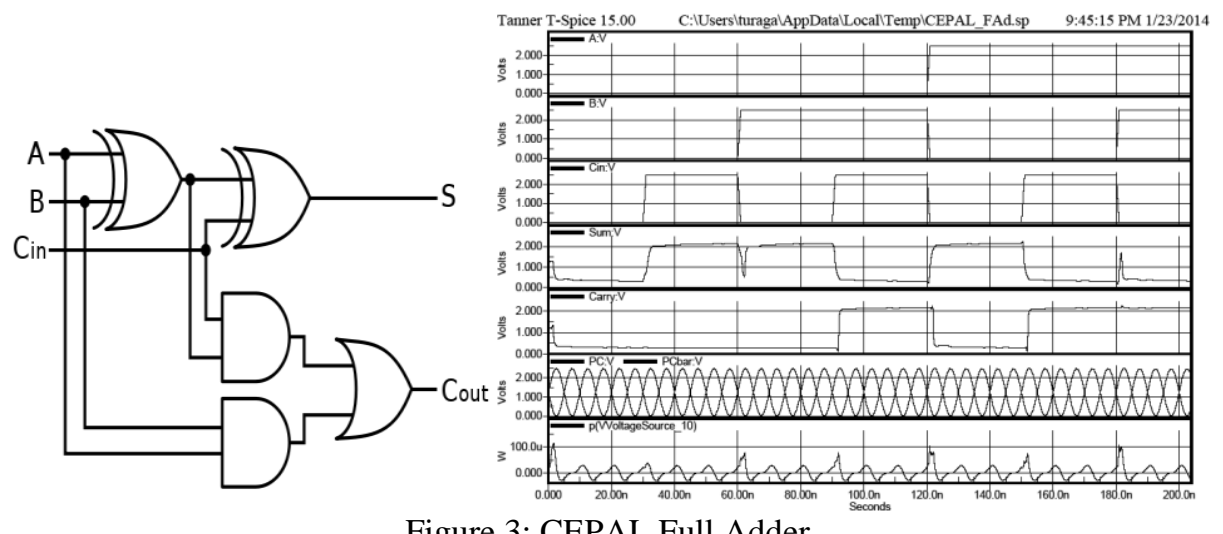

Figure 3: CEPAL Full Adder

IV. STRUCTURE AND DESIGN OF CEPAL MUX AND 4-BIT ALU

Arithmetic Logic Unit is a common operational unit with number of storage registers connected to it, using which it performs micro operations. To perform a micro operation, the contents of specified registers are placed in the inputs of the common ALU. The ALU performs an operation and the result of the operation is then transferred to a destination register. The ALU is a combinational circuit so that the entire registers transfer operation from the source register through the ALU and the destination register can be performed during one clock pulse period.

The ALU used here is the CEPAL ALU shown below in figure 4. It consist of two components the arithmetic unit and the logical unit.

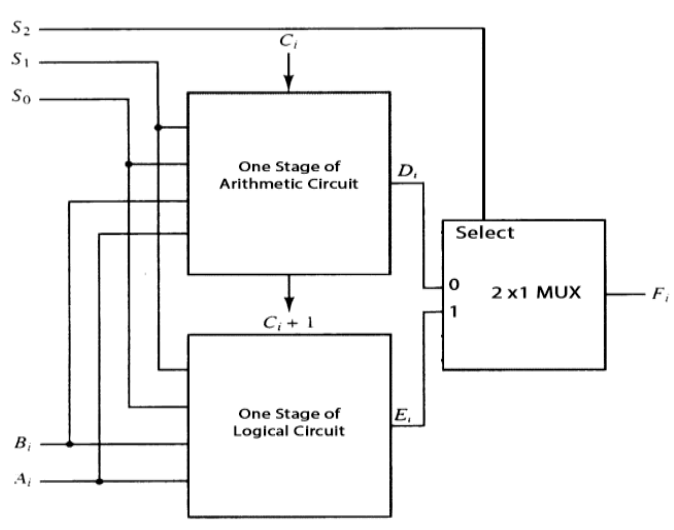

\begin{tabular}{ccccll}
\hline \hline \multicolumn{3}{c}{ Operation select } & & & \\
\cline { 1 - 3 }$S_{2}$ & $S_{1}$ & $S_{0}$ & $C_{\text {in }}$ & \multicolumn{1}{ll}{ Operation } & \multicolumn{1}{c}{ Function } \\
\hline 0 & 0 & 0 & 0 & $F=A$ & Transfer $A$ \\
0 & 0 & 0 & 1 & $F=A+1$ & Increment $A$ \\
0 & 0 & 1 & 0 & $F=A+B$ & Addition \\
0 & 0 & 1 & 1 & $F=A+B+1$ & Add with carry \\
0 & 1 & 0 & 0 & $F=A+\bar{B}$ & Subtract with borrow \\
0 & 1 & 0 & 1 & $F=A+\bar{B}+1$ & Subtraction \\
0 & 1 & 1 & 0 & $F=A-1$ & Decrement $A$ \\
0 & 1 & 1 & 1 & $F=A$ & Transfer $A$ \\
1 & 0 & 0 & $\times$ & $F=A \wedge B$ & AND \\
1 & 0 & 1 & $\times$ & $F=A \bigvee B$ & OR \\
1 & 1 & 0 & $\times$ & $F=A \oplus B$ & XOR \\
1 & 1 & 1 & $\times$ & $F=\bar{A}$ & Complement $A$ \\
AL & & & &
\end{tabular}

Figure 4: Structure of an ALU and its Truth Table 


\section{A. The Arithmetic Unit}

The arithmetic operations in the table can be implemented in one composite arithmetic circuit. The basic component of an arithmetic circuit is a full adder. By controlling the data input to the adder it is possible to obtain different types of arithmetic operations. The diagram of the 4-bit arithmetic circuit is shown in figure 5. It has four full adder circuits that constitute the 4 bit adder and 4 multiplexers for choosing multiple operations. There are two 4 bit inputs A and B and 4 bit output D.

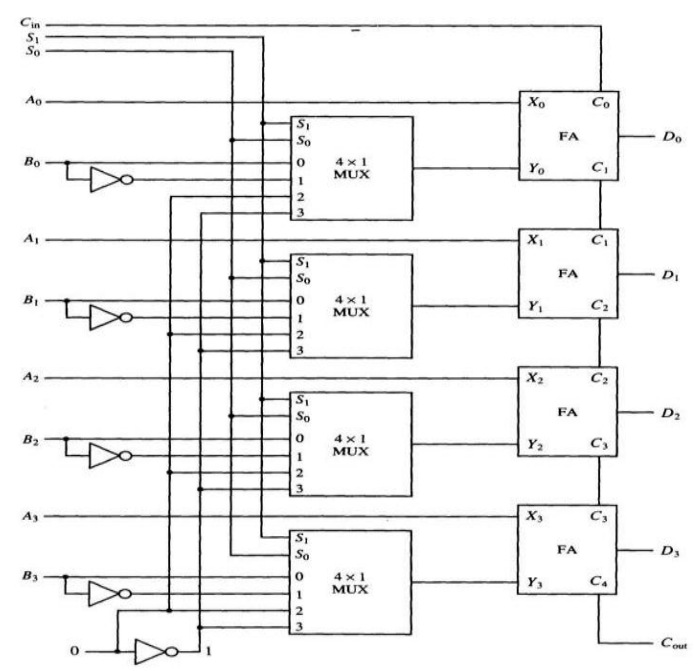

Figure 5: Schematic of 4-bit Arithmetic Unit

\section{B. Logical Unit}

Logic micro operations specify binary operations for strings of bits stored in registers. These operations consider each bit of registers separately and treat them as binary variables. Figure 6 shows one stage of a circuit that generates the four basic logic micro operations. It consists of 4 gates and a multiplexer each of the four logic operations is generated through a gate that performs the required logic. The outputs of the gates are applied to the data inputs of the multiplexer. The two selection inputs S1 and S2 choose one of the data inputs of the multiplexers and direct it values to the output. Figure 8 shows one typical stage of logical unit.

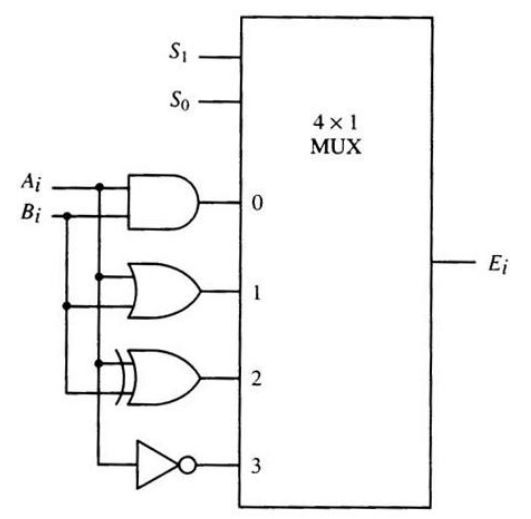

Figure 7: Schematic of 1-bit Logic Unit

\section{RESULTS AND DISCUSSIONS}

The adiabatic Arithmetic Logic Unit were implemented using 250nm technology of the Tanner EDA with a $\mathrm{W} / \mathrm{L}=0.35 \mathrm{um} / 0.24 \mathrm{um}$ and at $2.5 \mathrm{~V}$ operating voltage with power clock frequency at $100 \mathrm{MHz}$. The simulation results of both CMOS and CEPAL are compared with the same logic implementation. The simulation environment was maintained the same to provide justified results.

Figure 8 shows the output waveforms of the CEPAL Arithematic and Logic unit. The output clearly follows the truth tabe shown in figure 4 for the input bit sequence given is $\mathrm{A}=$ " 0011 " and $\mathrm{B}=$ " 1010 ". The $\operatorname{logic}$ ' 1 ' is $2.24 \mathrm{~V}$ and logic ' 0 ' is $0.18 \mathrm{~V}$. The plot $\mathrm{p}(\mathrm{VPC})$ show the power consumption of the power clock. 


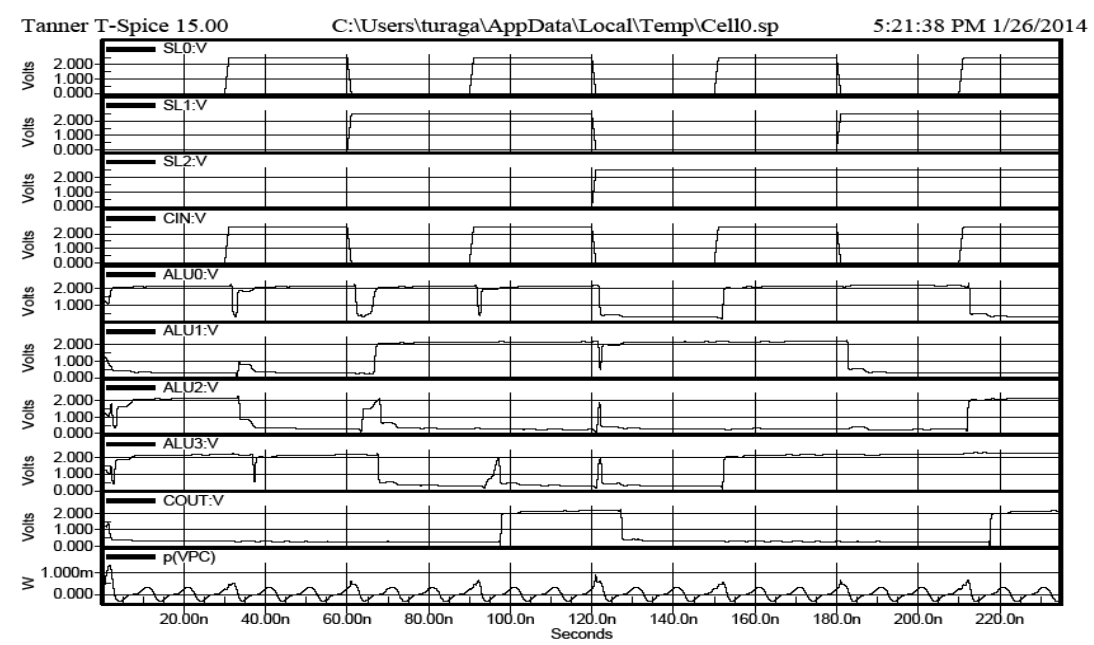

Figure 8: Output of CEPAL 4-bit ALU. in Table 8.

The number of transistors and the average power of the CMOS and the CEPAL 4-bit ALU are shown

Table 4: 4 bit ALU Transistor count \& Power Comparison

\begin{tabular}{|c|c|c|c|c|}
\hline Logic & P-MOS & N-MOS & Total Transistors & Average Power \\
\hline CMOS & 329 & 329 & 658 & $78.341 \mathrm{uW}$ \\
\hline CEPAL & 556 & 556 & 1112 & $35.723 \mathrm{uW}$ \\
\hline
\end{tabular}

\section{CONCLUSION}

The results clearly show that the CEPAL circuits offer better power efficiency compared to its CMOS counterpart. Though there is an increase in transistor count, it's very low power consumption outweighs this drawback. The simulation results show that the CEPAL ALU is 55\% more power efficient as compared to the CMOS ALU. CEPAL consumes significantly less power in contrast to CMOS in each and every case. CEPAL certainly does open many doors in design of low power VLSI circuits and is a promising alternative to the conventional CMOS in design of low power electronics. Its relatively high immunity to noise and increased throughput does facilitate its use in future electronics which is yet to come.

\section{ACKNOWLEDGEMENTS}

We are thankful to Dr. K. Manjunatha chari, Head of Department. Mrs. E. Arun Jyothi, Mr Md Masood Ahmad, Mrs. D Anitha, Mr.Govind Prasad, Mr N Shyam Sunder sagar, Dr. P. Trinatha Rao and all the other faculty members of the Department of Electronics and Communication, GITAM University - Hyderabad Campus for helping and guiding us toward the right path. We also thank Ms. Haritha Vellam for her support and faith in us.

\section{REFERENCES}

[1] Young moon and Deog Kyoon Jeong, "An Efficient Charge Recovery Logic Circuit," IEEE Journal of Solid State Circuits, Vol 31,No. 4, pp 514-522, April 1996.

[2] William C. Athas, Lars "JJ" Svensson, Jeffrey G. Koller, Nestoras Tzartzanis and Eric Ying-Chin Chou,"Low- Power Digital Systems Based on Adiabatic-Switching Principles," IEEE Transactions on Very Large Scale Integration (VLSI) Systems, Vol 2, No. 4, pp 398-407, Dec 1994.

[3] B. Dilli Kumar and M. Bharathi, "Design of Energy Efficient Arithmetic Circuits Using Charge Recovery Adiabatic Logic," International Journal of Engineering Trends and Technology, ISSN: 2231-5381, Vol:4, Issue 1, pp. 32-40, 2013.

[4] Ritu Sharma, Pooja Nagpal and Nidhi Sharma, "Analysis of Adiabtic NOR Gate for Power Reduction," International Journal of Latest Research in Science and Technology, ISSN (Online):2278-5299, Vol.1, Issue 2, pp 179-182, July-August 2012.

[5] B.Sravan Kumar, Rajeshwara Mahidhar.P and N.V.G.Prasad,"Energy Efficient Adiabatic Full Adders for Future SOC's," International Journal of Engineering and Advanced Technology (IJEAT),ISSN: 2249 - 8958, Volume-2, Issue-2, pp. 353-356, Dec 2012.

[6] Suresh Kumar Pittala, Swajeeth Pilot. Panchangam and A. Jhansi Rani, "Reliability Prediction for Low Power Adiabatic Logic Families," International Journal of Recent Technology and Engineering (IJRTE), ISSN: 2277-3878, Volume-1, Issue-3, pp. 116121, Aug. 2012.

[7] Sarita, Jyoti Hooda and Shweta Chawla, "Design and Implementation of Low Power 4:1 Multiplexer using Adiabatic Logic," International Journal of Innovative Technology and Exploring Engineering (IJITEE), ISSN: 2278-3075, Volume-2, Issue-6, pp. 224-227, May 2013

[8] A.Kishore Kumar, Dr.D.Somasundareswari, Dr.V.Duraisamy, and M.Pradeepkumar, "Low Power Multiplier Design Using Complementary Pass-Transistor Asynchronous Adiabatic Logic," International Journal on Computer Science and Engineering, Vol. 02, No. 07, pp. 2291-2297, 2010. 
[9] Mukesh Tiwari, Jai karan Singh and Yashasvi Vaidhya," "Adiabatic Positive Feedback Charge Recovery Logic for low power CMOS Design," International Journal of Computer Technology and Electronics Engineering (IJCTEE), ISSN 2249-6343, Volume 2, Issue 5, pp. 19-24, Oct. 2012

[10] Vijayasalini. P, Nirmal kumar. R, Dhivya. S. P and Dr. G.M. Tamilselvan, "Design and Analysis of Low Power Multipliers and 4:2 Compressor Using Adiabatic Logic," International Journal of Emerging Technology and Advanced Engineering, ISSN 2250-2459, ISO 9001:2008 Certified Journal, Volume 3, Issue 1, pp. 228-293, Jan. 2013.

[11] Monika Sharma, "Design and Analysis of CMOS Cells using Adiabatic Logic," International Journal of Networks and Systems, ISSN 2319 - 5975, Volume 1, No.2, pp. 52-57, Oct. - Nov. 2012.

[12] Nazrul Anuar, Yasuhiro Takahashi, and Toshikazu Sekine, "Two Phase Clocked Adiabatic Static CMOS Logic and its Logic Family," Journal of Semiconductor technology and Science, Vol.10, No.1, March 2010.

[13] Prasad D. Khandekar, Shaila Subbaraman, and Abhijit V. Chitre, "Implementation and Analysis of Quasi-Adiabatic Inverters", Proceedings of the International MultiConference of Engineers and Computer Scientists 2010, Vol II, IMECS 2010 , March 17 - 19, 2010, Hong Kong.

[14] Hamid Mahmoodi-Meimand and Ali Afzali-Kusha, Low-Power, "Low-Noise Adder Design with Pass-transistor Adiabatic Logic", The 12th International Conference on Microelectronics Tehran, Oct. 31 - Nov. 2, 2000.

[15] G.Rama Tulasi, K.Venugopal, B.Vijayabaskar, R.SuryaPrakash, "Design \& Analysis of full adders using adiabatic logic". International Journal of Engineering Research \& Technology (IJERT), ISSN: 2278-018., Vol. 1 Issue 5, July - 2012.

[16] Nazrul Anuar, Yasuhiro Takahashi, and Toshikazu Sekine,'Two Phase Clocked Adiabatic Static CMOS Logic and its Logic Family", Journal of semiconductor technology and science, Vol.10, NO.1, March, 2010.

[17] Amit Shukla, Arvind Kumar, Abhishek Rai and S.P. Singh, "Design of Low Power VLSI Circuits using Energy Efficient Adiabatic Logic,” International Journal of Scientific \& Engineering Research, ISSN 2229-5518, Vol. 4, Issue 6, pp. 349-358, June 2013.

[18] Shipra Upadhyay, R.A. Mishra and R. K. Nagaria,"Performance Analysis of Modified QSERL Circuit," International Journal of VLSI design \& Communication Systems (VLSICS), Vol.4, No.4, pp 19-30, Aug. 2013.

[19] Yibin Ye, And Kaushik Roy, "QSERL: Quasi-static Energy Recovery Logic", IEEE Journal of Solid-state Circuits, vol.36. no.2, pp. 239-248,Feburary.

[20] Gong, C.-S.A., Muh-Tian Shiue, Ci-Tong Hong, Chun-Hsien Su and Kai-Wen Yao, "Analysis and design of an efficient complementary energy path adiabatic logic for low-power system applications," SOC Conference, 2007 IEEE International, pp. 247 -251 , Sept. 2007

[21] Gong, C.-S.A., Muh-Tian Shiue, Ci-Tong Hong, Chun-Hsien Su and Kai-Wen Yao, "Analysis and design of an efficient Irreversible Energy Recovery Logic," IEEE Transactions on Circuits and Systems. Vol :55, No. 9, pp. 2595 - 2607 , Oct 2008.

[22] Shipra Upadhyay, R.A. Mishra, R.K. Nagaria, S.P. Singh and Amit Shukla "Triangular Power Supply Based Adiabatic Logic Family," World Applied Sciences Journal, ISSN 1818-4952, pp. 444-450, 2013.

[23] B.Nireesha, E.Mahender Reddy and S.Latha, "Simulation of Tree Adder Designed With Complementary Energy Path Adiabatic Logic," IOSR Journal of VLSI and Signal Processing (IOSR-JVSP), ISSN No. : 2319 - 4197, Volume 3, Issue 4, pp. 27-33, Nov. Dec. 2013.

[24] Sasipriya. p and Kanchana Bhaaskaran. V. S, "Single Phased Clocked Quassi Static Adiabatic Tree Adder," International Conference on Devices, Circuits and Systems (ICDCS), pp. 293-296, Apr 20, 2012. 